\title{
Radiation, Non-Ionizing DX or RX
}

National Cancer Institute

\section{Source}

National Cancer Institute. Radiation, Non-lonizing DX or RX. NCI Thesaurus. Code C18984.

Diagnostic or therapeutic use of non-ionizing radiation 